\title{
A solvable non-conservative model of Self-Organized Criticality
}

\author{
Gunnar Pruessner and Henrik Jeldtoft Jensen \\ Mathematics Department, Imperial College 180 Queen's Gate, London SW7 2BZ, United Kingdom \\ gunnar.pruessner@physics.org and h.jensen@ic.ac.uk
}

(June 1, 2018)

\begin{abstract}
We present the first solvable non-conservative sandpile-like critical model of Self-Organized Criticality (SOC), and thereby substantiate the suggestion by Vespignani and Zapperi [A. Vespignani and S. Zapperi, Phys. Rev. E 57, 6345 (1998)] that a lack of conservation in the microscopic dynamics of an SOC-model can be compensated by introducing an external drive and thereby re-establishing criticality. The model shown is critical for all values of the conservation parameter. The analytical derivation follows the lines of Bröker and Grassberger [H.-M. Bröker and P. Grassberger, Phys. Rev. E 56, 3944 (1997)] and is supported by numerical simulation. In the limit of vanishing conservation the Random Neighbor Forest Fire Model (R-FFM) is recovered.
\end{abstract}

PACS numbers: 64.60.Ht, 05.65.+b, 02.50.-r

The rôle of conservation in SOC-models is an old issue [1] 3] and is still unsettled. The number of nonconservative models which are definitely critical is, however, strikingly small. The Random Neighbor Forest Fire Model (R-FFM) [4 is one of them, while the Random Neighbor Olami-Feder-Christensen model (R-OFC) has been shown not to be critical in the non-conservative regime [5.6]. The nearest neighbor OFC model is commonly accepted to be critical in the conservative limit, but whether this model is critical in the non-conservative regime is still debated [2, [].

In [8,9] it has been suggested that non-conservation in the microscopic dynamics can be compensated by an external drive in order to re-establish criticality. Applying this concept directly to a model known to be non-critical in its original definition provides the ideal basis to identify the effect of such an external drive. In this letter such a model is defined and solved semi-analytically. The results are compared to simulations and the (trivial) critical exponents are extracted. Several limits are discussed.

The model, which is derived from the FFM 10 and the Zhang model 111, has three main parameters: $N$ is the total number of sites, which diverges in the thermodynamic limit. The number of randomly chosen "neighbors" is given by $n$, where $n=4$ in all examples, corresponding to a two dimensional square lattice. The conservation parameter is $\alpha$. The degree of nonconservation is then $1-n \alpha$, as it is shown below. Each site $i \in\{1,2, \cdots, N\}$ has associated a value $z_{i}$ for its "energy". Sites with $0 \leq z_{i}<1-\alpha$ are said to be "stable", sites with $1-\alpha \leq z_{i}<1$ are called "critical" and sites with $1 \leq z_{i}$ are "active". Negative energies are not allowed. The probability density function (PDF) for the variable $z_{i}$ is $P(z)$ with $z \in[0,1[$ and is defined only when no sites are active. $P(z)$ contains most of the stationary properties of the model.

The dynamics of the model are defined as follows: After an initial choice of $z_{i}(i=1,2, \ldots, N)$ from a uniform distribution in the interval $[0,1[$, the model is updated by repeatedly (i) "driving", (ii) "triggering" and (iii) "re- laxing" the system. During the drive (i), $i=1, \ldots,(1 / \theta)$ sites are chosen randomly $((1 / \theta)=p / f$ in the notation of [10]), one after the other, and their energy $z_{i}$ are set to $1-\alpha$, if the site is stable, otherwise $z_{i}$ remains unchanged. Subsequently one random site $j$ is chosen and if it is critical, the system is triggered (ii) by setting the energy of the chosen site to 1, i.e. making it active (initial seed). Otherwise $z_{j}$ remains unchanged and the model is driven again by repeating (i). As long as $N$ and $(1 / \theta)$ are finite, the system will escape from the driving loop sooner or later. In the thermodynamic limit this is ensured by a non vanishing density of critical sites.

During the relaxation (iii) the energy of each active site $i$ is redistributed according to the conservation parameter $\alpha$ to $n$ randomly chosen sites $j$ and the energy of $z_{i}$ is then set to 0 :

$$
z_{j} \rightarrow z_{j}+\alpha z_{i} \quad z_{i} \rightarrow 0
$$

Each visit or "toppling" (1) defines a microscopic time step and dissipates exactly $(1-n \alpha) z_{i}$ energy units. The sites $j$ are chosen randomly one after the other and are not necessarily different. In the thermodynamic limit the probability of choosing a target site which is already active or was already charged during the same avalanche, vanishes and therefore the order of these visits is irrelevant. In this very restricted sense the model might be considered as "Abelian". In contrast, sites in finite systems have always a finite probability to get charged more than once. Nevertheless, this probability decreases rapidly with increasing system size.

The number of active sites relaxed by (11) defines the avalanche size $s$, which is always positive due to the initial seed. In the stationary state the avalanches must dissipate, on average, the same amount of energy as is supplied by the external drive and the initial seed. The average dissipation depends on the avalanche size weighted average energy of active sites $\overline{z_{\text {act }}}$, which is equivalent to the average energy of active sites per toppling. Therefore

$$
(1-n \alpha) \overline{z_{\mathrm{act}}}\langle s\rangle=(1 / \theta) \frac{\overline{p_{\mathrm{st}}}}{\overline{p_{\mathrm{c}}}}\left(1-\alpha-\overline{z_{\mathrm{st}}}\right)+\left(1-\overline{z_{\mathrm{c}}}\right)
$$


must hold exactly in the stationary state even for finite systems and does not introduce any approximation. Here $\langle s\rangle$ is the average avalanche size, $\overline{p_{\mathrm{st}}}\left(\overline{p_{\mathrm{c}}}\right)$ is the density of stable (critical) sites (the drive stage is, on average, repeated $1 / \overline{p_{\mathrm{c}}}$ times), $\overline{z_{\mathrm{st}}}$ and $\overline{z_{\mathrm{c}}}$ are the average energy of stable and critical sites respectively. As in [5] the only crucial assumption is that $\langle s\rangle / N$ as well as $(1 / \theta) / N$ vanishes in the thermodynamic limit, which turns out to be entirely consistent with the results. This assumption allows us, for example, to assume the distribution $P(z)$ to be essentially unaffected by external drive or relaxation for sufficiently large systems.

From (2) it is clear that in general $\langle s\rangle$ diverges for diverging $(1 / \theta)$ or vanishing dissipation rate $1-n \alpha$. From the microscopic dynamics it is clear that there is always a non-vanishing fraction of sites with $z=0$, therefore $(1-\alpha)-\overline{z_{\mathrm{st}}}$ is finite and a divergence of $(1 / \theta)$ entails a divergence of $\langle s\rangle$, which is a sign of criticality.

In the following outline of the actual calculation, which is adapted from [5], the PDF's of the model are derived.

After an avalanche, each site belongs to one of $m+2$ classes, where $m=\lfloor 1 / \alpha\rfloor$. The index $k=0,1, \ldots, m$ of the class indicates the number of charges received from other toppling sites since their last toppling, while $k=$ $m+1$ indicates the class of sites, whose energy has been set by external drive. A site charged more than $m$ times must be active. For each of these classes a conditional distribution function $Q_{k}(z)$ is introduced, describing the distribution of energy among non-active sites, which have been charged $k=0, \ldots, m$ times or externally driven, $k=$ $m+1$. The distribution of sites which have not been charged since their last toppling, $Q_{0}(z)$, is a delta peak at $z=0$. For convenience the normalization of $Q_{0}(z)$ is chosen to be unity and all other distribution functions are normalized relative to class 0 . The distribution of sites, which have been driven externally and have not changed since then, $Q_{m+1}(z)$, is obviously a delta peak at $1-\alpha$. If the fraction of externally driven sites is $g, P(z)$ can be written as

$$
P(z)=\mathcal{N} \sum_{k=0}^{m+1}(1-g) Q_{k}(z)
$$

with appropriately chosen normalization $\mathcal{N}<1$. The upper bound for the energy of an active site is the geometric sum $1+\alpha(1+\alpha(\cdots))=1 /(1-\alpha)$, neglecting double charges. Therefore, the support of the distribution function of active sites $C(z)$ is $[1,1 /(1-\alpha)[$. If this distribution is normalized, the expected increase per avalanche in the class $k>0$ is given, in the thermodynamic limit (where multiple toppling can be neglected), by the convolution

$$
n\langle s\rangle \int_{1}^{1 /(1-\alpha)} d z^{\prime} C\left(z^{\prime}\right) Q_{k-1}\left(z-\alpha z^{\prime}\right),
$$

where the factor $n\langle s\rangle$ takes into account the expected total number of charges. There are three different ways in which the classes $k<m+1$ may be decreased:

1) By charges, $Q_{k}(z) n\langle s\rangle$

2) By external drive, $Q_{k}(z) \theta^{<}((1-\alpha)-z)(1 / \theta){\overline{p_{\mathrm{c}}}}^{-1}$, where $\theta^{<}$is the Heaviside unit function with $\theta^{<}(0)=0$. 3) By initial seed, $Q_{k}(z) \theta^{>}(z-(1-\alpha)) / \overline{p_{\mathrm{c}}}$, where $\theta^{>}(0)=$ 1 correspondingly.

Adding these contributions together and assuming stationarity leads to $m$ equations for $Q_{k}, k=1, \cdots, m$ :

$$
Q_{k}(z) l(z)=\int_{1}^{1 /(1-\alpha)} d z^{\prime} C\left(z^{\prime}\right) Q_{k-1}\left(z-\alpha z^{\prime}\right)
$$

where

$$
\begin{array}{r}
l(z)=1+\frac{(1 / \theta)}{\overline{p_{\mathrm{c}}} n\langle s\rangle} \theta^{<}((1-\alpha)-z)+ \\
\frac{1}{\overline{p_{\mathrm{c}}} n\langle s\rangle} \theta^{>}(z-(1-\alpha))
\end{array}
$$

has been used. For diverging $(1 / \theta)$, the last term in (6) becomes irrelevant and the RHS of (2) is dominated by the first term, meaning that the initial seed becomes irrelevant compared to the external drive. It is reasonable to restrict the range of $\alpha$ so that single charges cannot activate a site, $\alpha /(1-\alpha)<1 \Leftrightarrow \alpha<1 / 2$ (due to $n \alpha<1$, this is a restriction only for $n=1)$. Then one of the Eqs. in (5) can be written as

$$
C\left(\frac{z}{\alpha}\right)=\alpha Q_{1}(z) l(z)
$$

due to the particularly simple form of $Q_{0}(z)$.

Since $Q_{0}(z)=\delta(z)$ by definition and $\mathcal{N}(1-$ $g) Q_{m+1}(z)=g \delta((1-\alpha)-z)$ from (3), one further equation is necessary in order to find $m+3$ distributions $Q_{k}$, $k=0, \cdots, m+1$ and $C(z)$. This equation concerns the construction of the distribution of active sites $C(z)$. Since active sites are created due to charging or as the initial seed the average distribution of the number of those sites per avalanche is given by

$$
\langle s\rangle C(z)=n\langle s\rangle \int_{1}^{1 /(1-\alpha)} d z^{\prime} C\left(z^{\prime}\right) P\left(z-\alpha z^{\prime}\right)+\delta(z-1)
$$

where the $\delta$-function represents the initial seed.

Although it is a priori unknown whether there exists a stable solution, or whether it is unique, the set of equations given above is enough to start an iteration procedure in order to find a solution. The scalar parameters required are $\langle s\rangle$ from (2) $, \overline{p_{\mathrm{st}}}, \overline{p_{\mathrm{c}}}, \overline{z_{\mathrm{st}}}, \overline{z_{\mathrm{c}}}$, which are easily derived from (3) and $\overline{z_{\text {act }}}$, the first moment of $C(z)$. While $n$ and $(1 / \theta)$ parameterize the problem, $g$ remains the only unknown quantity, which is found to be

$$
g=\frac{\overline{p_{\mathrm{st}}}(1 / \theta)}{n\langle s\rangle \overline{p_{\mathrm{c}}}+1}
$$

by comparing the in- and outflow of class $m+1$, the externally driven sites, per avalanche. 
All the equations above can alternatively be derived directly from the fundamental dynamics. This ensures that the solution is exact in the thermodynamic limit given the stationarity assumption.

The implementation of the iteration procedure is straight-forward. As a criterion for termination, one could check whether $C(z)$, as defined by (8), is properly normalized [5], as it can be proven that it must be correctly normalized if it is a solution. However, it would be sufficient to assume $C(z)$ proportional to the RHS in (8). Moreover, in the numerical procedure the quality of the normalization of $C(z)$ depends strongly on the resolution of the grid chosen, whenever $C(z)$ changes rapidly as function of $z$. Therefore convergence of the iteration procedure is better verified by checking whether $C(z)$ approaches a fixed point, i.e. is invariant under (8). Since the distribution is expected to be highly non-analytic there are at least two $\delta$-functions in $P(z)$ - sophisticated integration routines are inappropriate. For $n=4$ the procedure quickly converges for $0.07<\alpha<0.24$, all nonpathological initial values tested lead to the same stable solutions. Only for small values of $\langle s\rangle$, when the $\delta$-peak of the initial seed starts to propagate through the distribution, a large grid is required for sufficient resolution. The same problem appears close to the commensurable limits mentioned below.

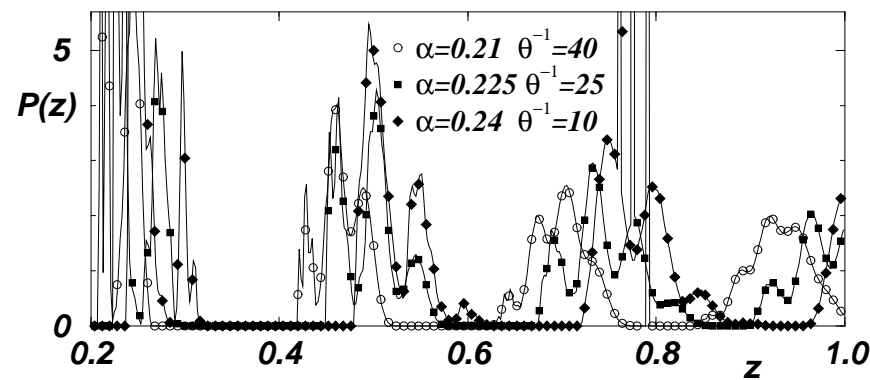

FIG. 1. Distribution of energy, $P(z)$, for $n=4$ and different values of $\alpha$. Since there is only a $\delta$-peak in $0 \leq z \leq 0.2$, results for $z<0.2$ are cut off. Continuous lines indicate results from theory (grid size 32000, integrated in 125 bins), points represent results from numerical simulations with $N=10^{6}$, $10^{6}$ avalanches for equilibration and $510^{6}$ avalanches for statistics (125 bins for the histogram).

In Fig. 1 numerical simulations of the model are compared to the numerical solution of the analytical approach. Although the PDF is very structured, discrepancies are small and can be reduced by increasing the resolution of the underlying grid.

The distribution $C(z)$ collapses to a $\delta$-function in at least two limits. Firstly, when $\alpha \rightarrow 0$, the FFM-limit, the probability for a site to become critical due to a number of charges vanishes as $q^{(1-\alpha) / \alpha}$, where $q<1$ is the product of the probability that a site receives a charge from a relaxing site and the probability that a site is not driven externally between two hits. Hence, for $\alpha \rightarrow 0$ the mechanism of "growing by charges" becomes negligible and the external drive becomes the dominating source for critical sites. The dynamics now become equivalent to the FFM: stable sites = empty sites, critical sites $=$ trees, and active sites $=$ fires. Furthermore, as $\alpha \rightarrow 0$, the support of $C(z)$ becomes smaller and smaller and the distribution of active sites is strongly peaked at $z=1$, collapsing to a $\delta$-function. Therefore, the distributions $Q_{k}, k>0$ are less smeared out, as shown in Fig. 2(a) for a small value of $\alpha$. Assuming $C(z)$ to be a $\delta$-function, one can easily reconcile the results in [4] (Eqs. (3) and (7)). The assumption that $P(z)$ is unaffected by single avalanches corresponds to $p, f \rightarrow 0$ in the SOC-limit of the FFM [12].

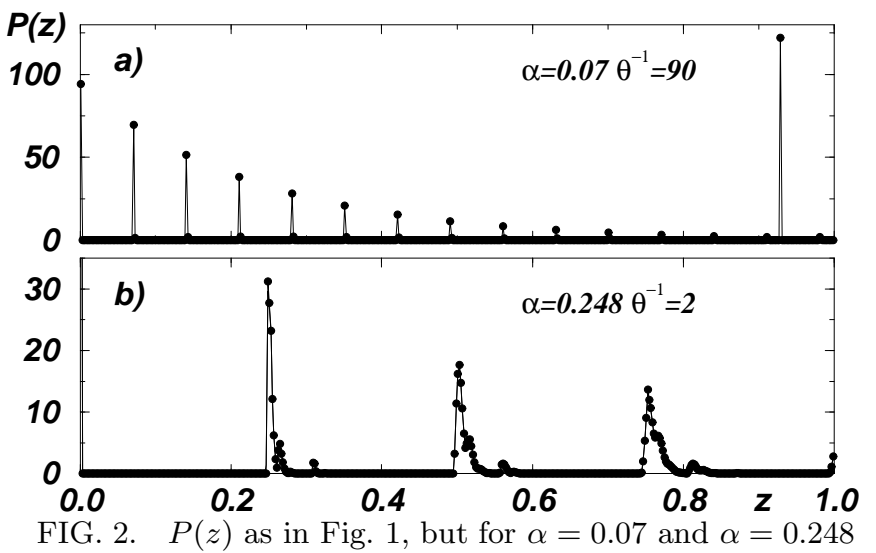
and 500 bins.

In the second limit, $\alpha \rightarrow 1 / n$, the model becomes conservative, but more important, $\alpha$ becomes more and more commensurable in the sense that a site charged $m=n$ times is almost always active and therefore the support of $Q_{m}$ vanishes, as it is squeezed between $n \alpha$ and 1 . When $\alpha$ is very close to $1 / n$, most of the active sites are provided by $Q_{m-1}$ and their average energy is just above 1 , i.e. $C(z)$ becomes more and more $\delta$ shaped and so do the $Q_{i}$, as shown in Fig. 2(b). The same behavior is obtained whenever $k \alpha=1$ for $k \in \mathbb{N}$.

The critical exponents of the model, can be obtained by mapping it on to a branching process [13] in order to identify the critical exponent $b=2$, where $\mathcal{P}(t) \propto t^{-b}$ is the exponent of avalanche duration. The exponent $\tau=3 / 2$, found by mapping the model on to a random walker along an absorbing barrier, is the exponent of avalanche sizes, $\mathcal{P}(s) \propto s^{-\tau}$. Formally these exponents arise only for diverging cutoffs in the distributions, which are controlled by the average number of active sites produced per single toppling, the branching ratio $\sigma$. The cutoffs diverge for $\sigma \rightarrow 1$.

However, the mapping is non-trivial, except when $C(z)$ is a $\delta$-function. This is because a distribution of active sites entails a distribution in the branching ratio, i.e. the branching probability itself becomes a random variable. 
This problem is removed by considering the ensemble average with an annealed rather than quenched disorder in the branching probability, i.e. writing the probabilities for one given node (active site) to branch into $k$ new nodes (active sites) as

$$
\mathcal{P}(1 \rightarrow k)=\left\langle\left(\begin{array}{l}
n \\
k
\end{array}\right) p^{k}(1-p)^{n-k}\right\rangle_{p}
$$

where $p$ denotes the branching probability (which is a function of the energy of the site) and \langle\rangle$_{p}$ denotes the weighted average over the probabilities. Therefore $\sigma=\sum_{k} k \mathcal{P}(1 \rightarrow k)$. This branching process is characterized by the same generating functions as the standard branching process [13], which becomes critical for $\sigma=1$. Hence the condition for criticality is

$$
\sum_{k=0}^{\infty} k\left\langle\left(\begin{array}{l}
n \\
k
\end{array}\right) p^{k}(1-p)^{n-k}\right\rangle_{p}=n\langle p\rangle_{p}=1
$$

which is the (average) branching ratio, according to (8) given by

$n\langle p\rangle_{p}=n \int_{1}^{1 /(1-\alpha)} d z \int_{1}^{1 /(1-\alpha)} d z^{\prime} C\left(z^{\prime}\right) P\left(z-\alpha z^{\prime}\right)=1-\frac{1}{\langle s\rangle}$.

Moreover $C(z)$ is time or generation dependent, since it evolves from an initial distribution which is only a $\delta$ function at $z=1$. The deviation of $C(z)$ from the final distribution decays exponentially fast, which can be seen by investigating the Markov chain of the repetitive convolution of a now time dependent $C(z)$ with $P(z)$ as in (8). Therefore the cutoff, introduced by the deviation of $\sigma$ from 1 , is dominated by the asymptotic iteratively stable limit of $C(z)$ only. Since the asymptote is approached exponentially fast the transient cannot influence the value of the exponents. The same arguments apply for the random walker approach, therefore $b=2$ and $\tau=3 / 2$ is true for all $\alpha \in] 0,1 / n[$.

The calculations above are a priori valid only in the thermodynamic limit. However, a simulation of the model must consider a finite system. Moreover the model relies on several assumptions, which entail certain finite size scaling: $(1 / \theta)_{N} / N$ (the index indicates the value to be measured in a system of size $N)$ as well as $\langle s\rangle_{N} / N$ must vanish for diverging $N$, while $(1 / \theta)_{N} /\langle s\rangle_{N}$ must remain constant. It is a well known problem in the FFM [14] that the number of trees grown between two ignitions is a parameter, $(1 / \theta)_{N}$, which needs to be tuned according to the system size; it is supposed to diverge, but its value is restricted by system size. An inappropriatly chosen parameter produces a small value of the cutoff or a bump in the distribution function of avalanche sizes. Nevertheless, $P(z)$ depends only weakly on $(1 / \theta)_{N}$. As a more quantitative measure for the "right choice of
$(1 / \theta)_{N}$ " we compared $g_{N}$ to $g$ (see Eq. (9)) in the thermodynamic limit. Assuming a cutoff of order $\mathcal{O}(N)$ in $\mathcal{P}(s)$ of a finite system, the scaling is $\langle s\rangle_{N}=\int d s \mathcal{P}(s) s \in$ $\mathcal{O}\left(N^{1 / 2}\right)$ and thus $(1 / \theta)_{N} \in \mathcal{O}\left(N^{1 / 2}\right)$. For a more quantitative picture one can map the avalanche on to a random walker along an absorbing barrier with time dependent walking probability $p(t)$ (in the sense of [15] a drinking rather than a drunken random walker). However, the result is comparatively involved and gives only rough estimates for the avalanche size as a function of $N$ and $(1 / \theta)$.

In summary, a solvable SOC model, critical in the entire regime of the conservation parameter, has been defined and the main properties have been derived. The critical exponents are as expected the trivial exponents of a critical branching process and a random walker. The model clarifies the rôle of the external drive and represents an explicit example of the recovery of criticality by introducing an external drive.

The authors acknowledge the support of EPSRC. G.P. wishes to thank Yang Chen and Richard Rittel for useful discussions as well as Quincy Thoeren for hospitality.

[1] G. Grinstein, D.-H. Lee, and S. Sachdev, Phys. Rev. Lett 64, 1927 (1990).

[2] A. A. Middleton and C. Tang, Phys. Rev. Lett. 74, 742 (1995).

[3] H. J. Jensen, Self-Organized Criticality (Cambridge University Press, New York, NY, 1998).

[4] K. Christensen, H. Flyvbjerg, and Z. Olami, Phy. Rev. Lett. 71, 2737 (1993).

[5] H.-M. Bröker and P. Grassberger, Phys. Rev. E 56, 3944 (1997).

[6] M.-L. Chabanol and V. Hakim, Phys. Rev. E 56, R2343 (1997).

[7] S. Lise and M. Paczuski, Phys. Rev. E 63, 036111 (2001).

[8] A. Vespignani and S. Zapperi, Phys. Rev. E 57, 6345 (1998).

[9] R. Dickman, M. A. Muñoz, A. Vespignani, and S. Zapperi, Braz. J. Phys. 30, 27 (2000), (condmat/9910454v2).

[10] B. Drossel and F. Schwabl, Phys. Rev. Lett. 69, 1629 (1992).

[11] Y.-C. Zhang, Phys. Rev. Lett. 63, 470 (1989).

[12] S. Clar, B. Drossel, and F. Schwabl, J. Phys. C: Condens. Matter 8, 6803 (1996).

[13] T. E. Harris, The Theory of Branching Processes (Springer, Berlin Göttingen Heidelberg, 1963).

[14] S. Clar, B. Drossel, and F. Schwabl, Phys. Rev. E 50, 1009 (1994).

[15] M. E. Fisher, J. Stat. Phys. 34, 667 (1984). 